\title{
VALORAÇÃO DE CONTINGENTE PELAS MODELAGENS LOGIT E ANÁLISE MULTIVARIADA: UM ESTUDO DE CASO DA DISPOSIÇÃO A ACEITAR COMPENSAÇÃO DOS CAFEICULTORES VINCULADOS AO PRO-CAFÉ DE VIÇOSA- MG ${ }^{1}$
}

\begin{abstract}
Pedro Silveira Máximo², Marcio Lopes da Silva e Maria Silveira Máximo ${ }^{4}$
RESUMO - O objetivo deste estudo foi, justamente, identificar, entre os métodos LOGIT e a análise multivariada, qual a mais eficaz para estimar a Disposição a Aceitar Compensação (DAC) dos cafeicultores quando o viés da utilidade marginal é passível de ocorrência. Para tal, foi elaborado um formulário com 33 perguntas envolvendo informações sobre características socioeconômicas dos cafeicultores, o uso da metodologia de valoração de contingente (MVC) e do veículo de pagamento dos "Jogos de Lances", que revelou a Disposição a Aceitar uma Compensação (DAC) na troca de um hectare de café por um hectare de mata. Como esperado, por causa do viés da utilidade marginal o método LOGIT foi incapaz de produzir resultados consistentes. Já a estimação da DAC pela análise multivariada mostrou que, caso o governo estivesse disposto a aumentar a provisão de mata em 70 ha, ele deveria despender 254.200 reais por ano, tratando apenas dos cafeicultores vinculados ao programa do PRO-CAFÉ.
\end{abstract}

Palavras-chave: LOGIT, análise multivariada e disposição a aceitar compensação (DAC).

CONTINGENT VALUATION WITH MODELING LOGIT AND MULTIVARIATE ANALYSES: A CASE STUDY OF THE WILLINGNESS OF COFFEE PLANTERS LINKED TO THE PRO - COFFEE OF VICOSA - MG TO ACCEPT COMPENSATION

\begin{abstract}
The object of this study was to identify which method, either LOGIT or multivariate analyses, was the most efficient to estimate the coffee planters' Willingness to Accept a Compensation, when there was a possibility of occurrence of marginal utility. For such, a questionnaire was formulated, with 33 questions involving information on coffee planters' socio - economic characteristics, the use of the methodology of contingent valuation (MCV), and the payment of the "offer game" that reveled the willingness to accept a compensation (WAC), by exchanging a hectare of coffee by a hectare of forest. As expected, because of the marginal utility's bias, the LOGIT method was unable to produce consistent results. However, when the WAC was estimated by multivariate analyses, the results showed that if the government is willing to increase the provision of forest to 70 hectares, it should pay out 254,200 reais (around 116,000 dollars), dealing only with the coffee planters linked to the PRO-CAFÉ organization.
\end{abstract}

Keywords: LOGIT, multivariate analyses and willingness to accept a compensation (WAC).

\section{INTRODUÇÃO}

A "valoração de contingente" é o método pelo qual o pesquisador tem a liberdade de criar um novo mercado e, assim, instituir as variáveis que julgar importantes para determinação do preço do recurso natural (COOPER, 2004).
A questão de se desenvolver um novo mercado passa por dois métodos de revelação de função de utilidade $^{1}$ da população: A Disposição a Pagar (DAP); e Disponibilidade de Aceitar Compensação (DAC). A Disposição a Pagar (DAP) pode ser compreendida como a máxima quantidade de renda que um indivíduo está

\footnotetext{
${ }^{1}$ Recebido em 30.10.2007 e aceito para publicação em 23.06.2009.

${ }^{2}$ Faculdade de Viçosa, FDV, Brasil.. E-mail: <pedro_ecoufv@yahoo.com.br>.

${ }^{3}$ Departamento de Engenharia Florestal da UFV, Viçosa, MG. E-mail: <marlosil@ufv.br>

${ }^{4}$ Graduação em andamento em Gestão de Cooperativas na Universidade Federal de Viçosa, UFV, Brasil.
} 
disposto a pagar pela troca de melhora das circunstâncias ambientais ou para evitar que elas piorem. De outra forma, é a quantidade de renda que compensa (equivale) aumento na oferta dos bens públicos, como é o caso da despoluição ou preservação de um rio. Já a Disposição a Aceitar Compensação (DAC) captura a mínima quantidade de renda que o indivíduo aceita para que as circunstâncias ambientais pelo menos não piorem, como foi o caso de Bishop e Heberlin (1979). Esses descobriram a disponibilidade dos caçadores em vender suas licenças de caça. Para Cooper et al. (2004), há distinção entre DAP e DAC: a primeira trabalha com uma hipotética provisão de bens públicos; a segunda com compensação para uma hipotética perda de bens públicos ou privados.

Usualmente, a DAC e DAP são determinadas pelo modelo econométrico que adota a distribuição do tipo LOGIT ${ }^{2}$. Entretanto, há outra forma ${ }^{3}$ de se obter o valor do recurso natural, que é sujeitar a amostra a uma análise multivariada, como a de Cluster e, posteriormente, recorrer à análise discriminante, estabelecendo, assim, uma desejada uniformidade dos dados e possibilitando inferências. No entanto, sentimentos relacionados ao recurso natural como afeição, valor de legado e tempo investido podem gerar comportamentos de difícil previsibilidade estatística. Problemas esses atribuídos à utilidade marginal ${ }^{4}$ do público-alvo da pesquisa. Por exemplo, por conta do tempo investido em determinado terreno, proprietários pobres podem exigir compensações financeiras relativamente superiores, quando comparado com os proprietários ricos.

Visto isso, por meio desses dois métodos esta pesquisa propôs valorar um recurso natural em determinado estudo de caso, no qual o viés da utilidade marginal se mostrou evidente. Com essa discussão, acredita-se estar contribuindo não só para elaboração de um modelo mais eficaz de precificação de bens sem mercado e formulação de políticas econômicas, como também para o desenvolvimento de maior arcabouço prático e teórico que facilite o pesquisador a antecipar possíveis obstáculos nos modelos estatísticos.

\section{MATERIAL E MÉTODOS}

\section{1. Área de estudo e coleta de dados.}

Viçosa está localizada na região conhecida como Zona da Mata. Possui população de 71.624 habitantes (população estimada para 2004), em que 7,81\% moram na zona rural. Sua área abrange cerca de $300.264 \mathrm{~km}^{2}$, cuja cobertura florestal natural representa 57.310 ha $(24,27 \%$ da área total), dos quais $24.184,80$ (10,24\%) correspondem às matas e 33.125,31 (14,03\%) a capoeiras (COELHO et al., 2005).

A escolha do tamanho da amostra concentrou-se na população dos integrantes do PRO-CAFÉ 5 de Viçosa, que totalizavam 82 cafeicultores. Apesar de o método proposto por Carvalho e Castro (2001) indicar que apenas 11 cafeicultores deveriam ser entrevistados, preferiram-se por entrevistar 60 cafeicultores - praticamente todo universo - , por se acreditar que essa base de dados poderia tornar as análises da amostra mais ricas. Os produtores questionados eram provenientes de 26 localidades, sendo, em sua maioria, do sexo masculino, da faixa etária dos 40 aos 49 anos (29,8\%) e com escolaridade até o ensino fundamental $(50,9 \%)$.

\subsection{Elaboração do Formulário}

Com o objetivo de facilitar a análise e aplicação do formulário, este foi dividido em quatro seções: a primeira incluiu dados pessoais dos entrevistados; a segunda preocupou em avaliar as principais fontes de renda do entrevistado e quais eram as atividades mais lucrativas dentro da propriedade; a terceira centrou-se na área ambiental e nos bens e serviços providos pela mata; e, por fim, a quarta seção centrou no valor da indenização por meio do método "jogos de Lances".

A pergunta que envolvia a escolha da indenização (DAC), no caso, foi operacionalizada com a intenção de aumentar a provisão dos Bens e Serviços de Ecossistemas (BSE), por exemplo: ar puro, proteção do lençol freático, polinização etc. Formalizando, para o cafeicultor foi proposta permuta de 1 ha de café plantado por 1 ha de mata nativa. Assim como fizeram Bishop e Heberlin (1979) com a licença para a caça, também se escolheu um substituto próximo para o bem ambiental, que aqui foi o café.

A escolha da DAC como mecanismo para a valoração de contingente, em vez da DAP, foi orientada por Bateman (1986), que comentou a inviabilidade de se determinar a disposição de um proprietário rural a pagar pela preservação de algo que ele acreditava que já estivesse compulsoriamente pagando. Comprovando isso, Guimarães (2003) relatou que a mesma situação se aplica aos produtores rurais de Viçosa, que consideraram a mata como um custo de oportunidade não aproveitado. 


\subsection{O Método dos "Jogos de Lances"}

O método dos "Jogos de Lances" (Bidding Games) foi escolhido como veículo de pagamento. A indicação desse procedimento seguiu sugestão de Silva (2003) e Mattos et al. (2007), por ser a forma mais adequada para fornecer informações sobre a DAC dos entrevistados, visto sua fácil aplicação.

A escolha dos lances delineou-se pelo produto substituto próximo escolhido por esta pesquisa, o café. O período dos valores a serem oferecidos foi determinado em termos de anuidade. Na Tabela 1 encontrou-se, segundo os técnicos da Empresa de Pesquisa Agropecuária de Minas Gerais (EPAMIG), os possíveis valores que os cafeicultores podem lograr, em média, com uma média bianual ${ }^{6}$ de 50 sacas por hectare.

Foram selecionados os lances, sendo o menor de $\mathrm{R} \$ 2.000,00$ e o maior de $\mathrm{R} \$ 9.000,00$ reais. Os lances foram arredondados para facilitar o cálculo do agricultor durante a aplicação do formulário, tendo como objetivo enriquecer a qualidade da amostra. No intervalo de $\mathrm{R} \$ 2.000,00$ a $\mathrm{R} \$ 9.000,00$, foram introduzidos os valores em reais de 3.000,00; 5.000,00; 7.000,00; e 8.000,00.

Cabe aqui mencionar que, de acordo com Haab e Macconnel (2002), quando o entrevistado disser "não" para todos os lances oferecidos, posteriormente a esse deve ser perguntado o porquê disso. Caso a justificativa do "não" para o valor oferecido tenha sido a luz de uma desconfiança do governo ou do projeto em si, ou algo parecido, essa negativa deverá ser considerada por protesto e descartada dos cálculos da DAC.

\subsection{Estimando o DAC pela modelagem do LOGIT}

A estimativa da Máximo Verossimilhança (MV) é usualmente utilizada para estimar os casos da distribuição do tipo LOGIT, uma vez que esta estimativa não necessita de que o desvio-padrão seja conhecido, ao mesmo tempo que não importa se o erro possui distribuição normal padrão ou logística. Ressaltando que os estimadores obtidos pelo MV são idênticos aos dos Mínimos Quadrados Ordinários, essa regra vale tanto para regressões simples quanto para múltiplas, em que, conforme se vai aumentando o tamanho da amostra, mais próximo se estará do verdadeiro (variância) $\sigma^{2}$ populacional (GUJARAT, 2002).

As variáveis T e $I_{j}$ representam, respectivamente, o tamanho da amostra e a resposta do entrevistado sobre a possibilidade de aceitar, ou não, a compensação financeira. A variável $I_{j}$ pode assumir dois valores: 1 ou 0. Caso o entrevistado aceite alguma compensação, toma-se $I_{j}$ igual a 1 ; caso contrário, $I_{j}$ será igual a 0 . A função de Máximo Verossimilhança para o LOGIT de relação linear pode ser escrita da seguinte forma:

$\ln L(\alpha, \beta \backslash y, z, t)=\sum_{j=1}^{T} I_{j} \ln \left[1+e^{-\left(\frac{\alpha z_{j}}{\sigma}+\frac{\beta t_{j}}{\sigma}\right)^{-1}}\right]+\left(1-I_{j}\right) \ln \left[1-\left(1+e^{-\left(\frac{\alpha z_{j}}{\sigma}+\frac{\beta t_{j}}{\sigma}\right)}\right)^{-1}\right]$

Visto como estimar os parâmetros pela MV, a estimativa da disposição a aceitar pode ser obtida pela diferença entre o status quo (estado em que o entrevistado se encontra antes de o projeto ser realizado) e estado de preferência no final do projeto, como se vê a seguir:

$\alpha_{1} z_{j}+\beta\left(y_{j}+D A C_{j}\right)+e_{1 j}=\alpha_{0} z_{j}+\beta y_{j}+e_{0 j}$

Resolvendo a equação, tem se:

$D A C=\alpha z_{j} / \beta+e_{j} / \beta$

Todavia, para finalmente chegar à forma final existem três possíveis limitações:

i. Incerteza sobre a aleatoriedade das preferências: se $e_{j} / \beta$ possui média zero e a variância é $\sigma^{2} / \beta^{2}$.

ii. Incerteza sobre aleatoriedade dos parâmetros.

iii. Variação através dos indivíduos dentro de uma amostra: a expressão DAC pode ser calculada

Tabela 1 -Qualidade do café e sua rentabilidade.

Table 1 - Quality of coffee and its profitability.

\begin{tabular}{|c|c|c|c|}
\hline Qualidade do Café & Preço Médio $(\mathrm{R} \$)$ & Sacas (ha) & Lucro Líquido Anual (R\$) \\
\hline Baixa Qualidade & 70 & 50 & 2000,00 \\
\hline Media Qualidade & 160 & 50 & 4000,00 \\
\hline Boa Qualidade & 240 & 50 & 6000,00 \\
\hline Excelente Qualidade & 360 & 50 & 9000,00 \\
\hline
\end{tabular}

Fonte: EPAMIG

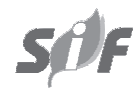

R. Árvore, Viçosa-MG, v.33, n.6, p.1149-1157, 2009 
para qualquer uma das variáveis independentes e, ainda, ser calculada para amostra inteira.

Tratando dessas limitações, os parâmetros são tidos como dados, viabilizando, assim, que medidas de tendência solucionem essas restrições. Mais especificamente, de acordo com Silva (2003) e por meio da tendência central, é possível obter a "verdadeira disposição a aceitar" dos entrevistados. Para tanto, uma dessas medidas é dada pela média:

$$
E_{e}\left(D A C_{j} \backslash \alpha, \beta, z_{j}\right)=\alpha z_{j} / \beta
$$

A segunda medida de tendência central é a mediana, capaz de capturar $50 \%$ da distribuição de uma função de preferência que sofre as limitações com incerteza. Essa medida de tendência central deve ser a mais utilizada dentro dos trabalhos de valoração de contingente, dado que os resultados se apresentam mais consistentes com a realidade. Isso foi verificado por meio de estudos comparativos realizados por Hanemann (1984).

$\operatorname{Md}_{e}\left(D A C_{j} \backslash \alpha, \beta, z_{j}\right)=\alpha z_{j} / \beta$

\subsection{Análise de Cluster e Discriminante}

\section{- Análise de Cluster}

O objetivo da Análise de Cluster é agrupar os elementos amostrais em g's grupos distintos, de forma que os elementos pertencentes a um grupo sejam os mais similares entre si. Dessa forma, considera-se que para cada elemento amostral, $\mathrm{j}$, tem-se um vetor de medidas, $\mathrm{Xj}$, com $\mathrm{p}=$ variáveis armazenadas (MINGOTE, 2005).

$X_{j}=\left[X_{1 j}, X_{2 j}, \ldots, X_{p j}\right] j=1,2,3, \ldots, n$

em que $X_{i j}$ representa o valor observado da variável $i$ medida no elemento amostral $j$.

Para proceder à transformação dos dados em grupos, é preciso que se decida "a priori” a medida de similaridade (ou dissimilaridade) que será adotada (MINGOTE, 2005). Entre as medidas de distância, uma das mais conhecidas é a euclidiana (Teorema de Pitágoras).

No caso da partição da amostra de cafeicultores em grupos, a técnica não hierárquica das K-médias foi escolhida. Tal procedimento está embasado em Krebs (1994) e Souza e Souza (2006). Eles comentaram que, além de essa técnica determinar um número ideal de partição, dois requisitos básicos são levados em consideração: maior coesão interna e menor dispersão interna (variância) dos dados dentro dos grupos; maior isolamento possível entre os clusters gerados, isto é, cada grupo formado possui características distintas um do outro.

Por meio de consultorias com técnicos do IPEA, notou-se que essa menor dispersão interna foi responsável por viabilizar inferências seguras sobre o comportamento dos produtores rurais do PROCAFÈ, como escolaridade e tamanho da propriedade, entre outras variáveis estudadas.

\section{- Análise Discriminante}

Contudo, como saber se a alocação da análise do K-médias foi realmente boa? E ainda, se ela não foi boa o suficiente, como melhorá-la? A resposta para isso é dada pelo uso da Análise Discriminante (AD). Pode-se entender a AD como uma técnica utilizada para classificação de elementos de uma amostra ou uma população, e, assim como nos modelos não hierárquicos, é necessário que os grupos estejam predefinidos, procedimento que permite a elaboração de uma função matemática (MINGOTE, 2005).

Supondo que o objetivo deste método é de justamente minimizar a probabilidade de erros (classificações incorretas), assumir-se-á um vetor fixo de observações, $x$, em que cada elemento amostral deverá ser alocado para a função distribuição (população k) que tiver maior probabilidade de ocorrência:

$$
f_{k}(x)=\text { máximo }\left\{f_{1}(x), i=1,2, \ldots, g\right\}
$$

Em que $g$ representa o numero de clusters e $f(x)$, a função-densidade.

De acordo com Mingote (2005), no caso em que o vetor aleatório $X_{j}$ tenha distribuição normal, com pvariáveis, essa regra é equivalente a classificar o elemento com o vetor observado $x$, na população $k$, de forma que:

$$
\begin{aligned}
& d_{k}^{Q}(x)=\text { máximo }\left\{d_{1}^{Q}(x), d_{2}^{Q}(x), \ldots, d_{g}^{Q}(x)\right\} \\
& \text { sendo: } \\
& d_{k}^{Q}(x)=-\frac{1}{2} \ln \left(\left|\sum_{i}\right|-\frac{1}{2}\left(x-\mu_{1}\right)^{\prime} \sum_{i}^{-1}\left(x-\mu_{i}\right)\right)
\end{aligned}
$$

os coeficientes quadráticos dados por $Q$, em que $\mu$ e $\sum$ representam, respectivamente, o vetor média e a matriz de covariância amostral da população $i=$ $1,2, \ldots, g$, sendo essa função conhecida como função discriminante de Fisher. 
No caso desta pesquisa, o número de cluster formado pela técnica do K-médias, que por sinal foram 4 (sugestão do IPEA), é que se delineou o número de funções discriminantes encontradas (MINGOTE, 2005). A partir dos 4 cluster formados, a análise discriminante desenvolverá quatro funções discriminantes, determinando os escores (ou coeficientes) de discriminação de cada uma das variáveis selecionadas como boas discriminadoras, e, ainda, para cada grupo se determina uma constante.

A análise da qualidade do ajuste da função discriminante é dada pelo teste de F multivariado (MINGOTE, 2005). Portanto, espera-se que os escores encontrados para um cluster sejam bem diferenciados dos obtidos de um cluster para outro cluster; essa comparação pode ser feita pelo teste de Hotelling, definido por:

$F=\frac{\left(n_{1}+n_{2}+\ldots+n_{g}\right)-p-1}{p\left(n_{1}+n_{2}+\ldots+n_{g}\right)} T^{2}$

em que:

$T^{2}=\frac{n_{1} n_{2} \ldots n_{g}}{n_{1}+n_{2}+\ldots+n_{g}}\left(\bar{Y}_{1}-\bar{Y}_{2}-\ldots-\bar{Y}_{g}\right)$

em que $p$ representa o número de populações e $\bar{Y}_{g}$ o g-ésimo escore da função discriminante, quando aplicada aos vetores de médias amostrais do cluster $g$. De acordo com Mingote (2005), essa função possui distribuição de F; e nesse caso possui $n_{1}+n_{2}-p-1$ graus de liberdade e nível de significância fixo. Em que $p$ representa o número de populações e $\bar{Y}_{g}$ o $g$-ésimo escore da função discriminante, quando aplicada aos vetores de médias amostrais do cluster $g$.

Quando p > 1, a comparação das médias populacionais dos escores da função discriminante dos grupos é feita pelo teste de Wilks de análise de variância multivariada, em que, segundo Mingote (2005), uma variável será considerada significativa para estar dentro da função discriminante até $15 \%$ de confiança. Já a retirada de uma variável qualquer do modelo será aceitável até o nível de significância de $25 \%$.

\section{RESULTADOS E DISCUSSÕES}

\subsection{Distribuição da Amostragem}

Segundo a Tabela 2 , apenas pequena parcela declarou não ter instrução $(8,77 \%)$, em que também se observaram que $60 \%$ dos entrevistados possuíam educação até o ensino fundamental. Com relação aos $40 \%$ restantes, com nível educacional mais elevado é justificado pela proximidade do campus da UFV com a zona rural e pelo aumento significativo do número de grupos escolares na região nos últimos 30 anos.

\subsection{Análise da Correlação entre DAC com os Bens e Serviços de Ecossistemas (BSEs), a Idade e a Renda dos Proprietários Rurais}

Segundo a matriz de correlação, reforçada pelo teste bicaudal de Spearman, notou-se relação positiva $(0,268)$ entre a escolaridade e o conhecimento da importância do serviço de polinização, e como também foi possível perceber relação inversa $(-0,347)$ entre a idade e o número de pessoas que declararam conhecer a capacidade da mata nativa em fazer o controle de pragas na lavoura de café. Essa realidade permite argumentar que os proprietários mais novos são mais conhecedores dos BSE providos pela natureza.

Por meio da matriz de correlação, notou-se a relação positiva entre a DAC e o preço da saca de café $(0,287)$, bem como a relação inversa entre a renda e a DAC (0,070). Para a ultima, à justificativa provavelmente se concentrou na própria teoria da utilidade marginal, dado que o acréscimo de 1 ha na propriedade do pequeno cafeicultor tem muito mais relevância do que esse mesmo acréscimo na propriedade de grande cafeicultor. Trazendo para a realidade dessa pesquisa, a perda de 1 ha de café teria muito mais peso para um pequeno proprietário do que para um grande.

\subsection{DAC dos cafeicultores estimada pelo modelo LOGIT}

Por meio da estimativa dos MQO, os resultados revelaram regressão com variáveis explicativas pouco significativas, haja vista o baixo valor de $\mathrm{R}^{2}$ e o fato de apenas a variável escolaridade mostrar-se representativa no nível de significância de 10\% (Tabela 2). Portanto, variáveis como renda familiar, idade, tamanho da propriedade (TDP), assim como os BSE, por explicarem pouco o comportamento da DAC dos cafeicultores, devem ser desconsideradas das análises.

Acompanhando as análises realizadas pelo modelo de MQO, o modelo com distribuição logística mostrou a inviabilidade de ser realizar qualquer estimava consistente sobre o valor da "verdadeira" DAC para preservar os fragmentos, dado que nenhuma das variáveis predeterminadas apresentaram significância inferior a 30\%.

R. Árvore, Viçosa-MG, v.33, n.6, p.1149-1157, 2009 
Tabela 2 -Regressão Múltipla entre a DAC dos cafeicultores e as variáveis explicativas pelos Modelos dos MQO e LOGIT. Table 2 - Multiple Regression between the DAC and the coffee growers of the explanatory variables and models of MQO LOGIT.

\begin{tabular}{|c|c|c|c|c|}
\hline \multicolumn{5}{|c|}{ Variável Dependente: DAC (por MQO) } \\
\hline Variável Independente & Coeficiente & Desvio Padrão & Sig (teste de T) & $\mathrm{R}^{2}$ \\
\hline BSE & 250.485 & 357.688 & 0.700 & 0.105 \\
\hline Escolaridade & 172.034 & 94.148 & 0.827 & Teste de F \\
\hline Idade & -13.689 & 31.620 & 0.093 & 1.1923 \\
\hline Renda & -0.112 & 0.217 & 0.517 & Durbin Watson \\
\hline TDP & -3.579 & 7.099 & 0.5042 & 2.52 \\
\hline Constante & 2467.809 & 2742.374 & 0.8998 & \\
\hline \multicolumn{5}{|c|}{ Variável Dependente: DAC (por Logit) } \\
\hline Variável Independente & Coeficiente & Desvio Padrão & Sig (teste de Z) & Previsões Corretas \\
\hline BSE & -0.013 & 0.277 & 0.748 & 97,556 \\
\hline Escolaridade & 0.019 & 0.073 & 0.868 & McFadden $\mathrm{R}^{2}$ \\
\hline Idade & 0.007 & 0.025 & 0.353 & 0,014 \\
\hline Renda & 0.000 & 0.000 & 0.400 & \\
\hline TDP & 0.005 & 0.005 & 0.767 & \\
\hline Constante & -1.454 & 2.159 & 0.674 & \\
\hline
\end{tabular}

Fonte: Dados da Pesquisa.

\subsection{Análise de Cluster}

A técnica das k-médias encontrou, para quatro clusters, apenas seis variáveis consideradas boas separadoras de grupos, em que, segundo a análise ANOVA, todas foram significativas até 5\%, sendo elas: DAC, Escolaridade, Filhos Trabalhando, Preço da Saca e Área Total da Propriedade. Cabe, agora, mostrar o tamanho de cada cluster formado e as suas principais características.

\section{Cluster 1}

Composto por apenas duas pessoas, menos de $4 \%$ da amostra, o cluster 1 caracterizou-se por ser o conglomerado com menor número de elementos e o mais rico entre os estudados. Os cafeicultores que compuseram esse grupo possuíam em comum o fato de terem cursado até o terceiro grau, de não terem nenhum filho trabalhando na propriedade e de apresentarem uma renda mensal superior a 10.000,00 reais. Eles, ainda, pediram valores semelhantes para indenização da perda de 1 ha de café, entre $\mathrm{R} \$ 4.000,00$ e $\mathrm{R} \$ 3.000,00$. Quanto às outras variáveis estudadas, os valores não foram tão próximos. O valor de venda da saca do café foi em torno de $\mathrm{R} \$ 215,00$ a $\mathrm{R} \$ 300,00$, e o tamanho da propriedade diferenciou em 19 e 28 ha, respectivamente.

Com base nessas informações, nenhuma medida segura sobre esse perfil de cafeicultor será adotada, entendendo que esse cluster formado capturou os outliers, o que também impossibilitou qualquer análise generalizada.

R. Árvore, Viçosa-MG, v.33, n.6, p.1149-1157, 2009

\section{- Cluster 2}

O cluster 2 caracterizou-se por ser o conglomerado mais populoso entre os formados, contendo 27 cafeicultores (ou 50\% da amostra) e também por ser o mais pobre, com renda média de $1.109,00$ reais por mês $(\mathrm{R} \$ 812,64)^{1}$. As principais características desse cluster foram: baixa escolaridade, 5,7 anos (5,06 anos) em média na escola; a maior média de filhos trabalhando na propriedade, $0,89(1,12)$; tamanho médio da propriedade de 21,756 ha (17,91 ha); e preço da venda da saca de café, 192,00 reais ( $\mathrm{R} \$ 54,180)$, em média.

Quanto à DAC, o cluster 2 mostrou que, em média, os cafeicultores estvam dispostos a receber 1.740 reais ( $\mathrm{R} \$ 1.227,40)$ por ano, ou $\mathrm{R} \$ 145,00$ por mês, como indenização para efetivarem a permuta entre mata e café. Entretanto, quando descontados os protestos (3), esse valor subiu para 1.958 reais $(R \$ 1.122,02)$ por ano, ou $\mathrm{R} \$ 163,33$ por mês. Como medida mais robusta a ser considerada, a mediana mostrou que os cafeicultores do cluster 2 aceitavam 2.000,00 reais por ano, ou $\mathrm{R} \$ 166,00$ por mês, mesmo com a retirada dos protestos. Algo que esse conglomerado permitiu, dado o seu tamanho, foi estimar uma regressão múltipla, sendo a DAC utilizada como variável dependente. Já a renda, filhos trabalhando na propriedade e escolaridade funcionaram como variáveis explicativas. As variáveis área total e preço da saca foram retiradas da análise por serem não significativas e estarem enviesando a análise geral do modelo. 
Considerando as informações da Tabela 3, é possível verificar que o acréscimo de um filho trabalhando na propriedade leva o cafeicultor a aumentar a sua DAC em 808,00 reais. Entende-se com isso que, quanto maior o número de filhos trabalhando na propriedade do produtor do cluster 2, maior a sua DAC, relação essa explicada pelo valor de legado, isto é, vontade do "pai" de deixar renda extra para o filho e maior área de mata.

Como explicado, a renda tem relação negativa com a DAC, indicando que 1 real a mais na renda implica diminuição da DAC em 0,899. Sobre a escolaridade, a regressão indicou que o aumento de um ano letivo irá fazer que o produtor aumente sua DAC em praticamente 174,413 reais. Por fim, o teste $\mathrm{F}$ indicou que a regressão estimada é globalmente válida, e o $\mathrm{R}^{2}$ mostrou que as variáveis explicativas foram capazes de explicar $60 \%$ do comportamento da DAC.

\section{- Cluster 3}

Com população relativamente pequena, seis cafeicultores, representando apenas $11,11 \%$ da população estudada, o cluster 3 caracterizou-se por ser o segundo mais rico, justificando a proximidade com o cluster 1. Em média, esses cafeicultores ganhavam mensalmente $5.416,00$ reais $(\mathrm{R} \$ 1.281,27)$ por mês, e, como era de se esperar, as médias do tempo na escola de 13,5 anos (3,46 anos), o tamanho da propriedade de 47 ha (25 ha), o preço da saca de 225,00 reais $(\mathrm{R} \$ 65,00)$ e o número médio de filhos trabalhando $0,16(0,4)$ foram todos relativos a proprietários com nível de renda maior.

Assim como no cluster 1 , julgou-se que qualquer análise perante a DAC desses cafeicultores seria arriscada e que havia necessidade de entrevistar maior número de cafeicultores com esse perfil para que qualquer análise segura pudesse ser feita, impossibilitando também estimar uma regressão sobre a DAC.

\section{- Cluster 4}

Com 19 cafeicultores (ou 35\% da amostra) e a segunda pior média de renda observada, $1.389,47$ reais por mês ( $\mathrm{R} \$ 960,26)$, o cluster 4 destacou-se por ser conglomerado mais bem-sucedido que os cafeicultores do cluster 2. Contudo, esse destaque não está diretamente relacionado com o fato de a renda do cluster 4 ser superior à do cluster 2 . Isso porque, pelo teste de " $t$ ", em duas amostras independentes constatou-se que as rendas dos clusters 2 e 4 eram estatisticamente iguais, dado que a significância do teste foi de $29,4 \%$, sendo o limite confiável para descartar a hipótese de igualdade de apenas $10 \%$. Portanto, credita-se a esse destaque do cluster 2 perante o 4 o fato de que, mesmo com tamanho médio da propriedade de 17,9 ha ( $15,70 \mathrm{ha})$, inferior ao observado no cluster 2 , esses produtores conseguiram retirar valor médio de venda da saca de café superior a 232,68 reais $(\mathrm{R} \$ 53,47)$. Outra diferença substancial entre esses dois clusters foi na escolaridade. No cluster 4, estudaram-se, em média, dois anos a mais que no cluster 2. Isto é, em média os cafeicultores do cluster 4 ficaram oito anos (5,06 anos) na escola; e, mais ainda, o número de filhos trabalhando no cluster 4 foi menor do que o verificado no cluster 2 , sendo em média $0,26(0,56)$ filho trabalhando por propriedade.

O valor da indenização aceito pela perda de 1 ha de café foi o maior observado entre todos os clusters formados. Verifica-se, assim, que, em média, os proprietários do cluster 4 estavam dispostos a aceitar 6.000,00 reais por ano, ou $\mathrm{R} \$ 500,00$ mês.

A regressão apresentada na Tabela 4 mostrou que um filho a mais trabalhando na propriedade induziu o proprietário a aumentar o valor da DAC em 1.248,10 reais. Um ano a mais na escola fez que o proprietário aumentasse a DAC em 78,43 reais.

Tabela 3 - Regressão Múltipla do Cluster 2.

Table 3 - Multiple Regression of Cluster 2.

\begin{tabular}{lcccc}
\hline \multicolumn{5}{c}{ Variável Dependente: DAC (por MQO) } \\
\hline Variável Independente & Coeficiente & Desvio Padrão & Sig. (Teste de t) & $\mathrm{R}^{2}$ \\
\hline Intercepto & 1024.506 & 454.936 & 0.034 & 0,60 \\
Filhos Trabalhando & 808.002 & 285.902 & 0.010 & Sig (Teste de F) \\
$\quad$ Renda & -0.899 & 0.371 & 0.024 & 0,043 \\
Escolaridade & 174.413 & 62.166 & 0.010 & Durbin Watson \\
& & & 2,361 & \\
\hline
\end{tabular}

Fonte: Dados da Pesquisa. 
Tabela 4-Regressão Múltipla do Cluster 4.

Table 4-Multiple Regression of Cluster 4.

\begin{tabular}{ccccc}
\hline \multicolumn{5}{c}{ Variável Dependente: DAC (por MQO) } \\
\hline Variável Independente & Coeficiente & Desvio Padrão & Sig. (Teste de t) & $\mathrm{R}^{2}$ \\
\hline Intercepto & 5808,4 & 681,118 & 0,000 & 0,505 \\
Filhos trabalhando & 1248,1 & 597,518 & 0,054 & Teste de F \\
Renda & $-0,5122$ & 0,3432 & 0,116 & 0,098 \\
Escolaridade & 78,4385 & 66,7708 & 0,258 & Durbin Watson \\
& & & 1,92 & \\
\hline
\end{tabular}

Fonte: Dados da Pesquisa.

Tabela 5 - Funções Discriminantes para cada cluster. Table 5 - Discriminants Functions for each cluster.

\begin{tabular}{lcccc}
\hline & \multicolumn{4}{c}{ Escores discriminantes de Cada Cluster } \\
\hline & 1 & 2 & 3 & 4 \\
Área total & $-0,184$ & 0,031 & 0,036 & $-0,049$ \\
Filhos Trabalhando & $-6,089$ & 0,398 & $-2,606$ & $-1,911$ \\
Renda & 0,015 & 0,001 & 0,007 & 0,003 \\
DAC & 0,005 & 0,001 & 0,002 & 0,004 \\
(Constante) & $-86,784$ & $-3,416$ & $-21,533$ & $-16,182$ \\
\hline
\end{tabular}

Fonte: Dados da Pesquisa.

Da mesma forma como ocorreu com o cluster 2, o aumento de 1 real na renda fez que o proprietário diminuísse a DAC em 0,512 reais, porém esse valor não é tido como representativo, visto que a renda não foi considerada variável representativa em um nível de significância de $10 \%$. O teste F considerou essa regressão globalmente representativa, e o $\mathrm{R}^{2}$ informou que as variáveis explicativas conseguiram explicar o comportamento de $50 \%$ da DAC.

\subsection{Análise discriminante}

A análise discriminante baseou-se nas mesmas seis variáveis escolhidas pelo teste ANOVA. No entanto, apenas quatro dessas foram consideradas significativas para discriminar a amostra: renda, DAC, filhos trabalhando e área total, em que somente a inclusão da renda poderia gerar certa dúvida, dado que a sua significância superou o limite recomendado de $15 \%$. O teste F e a tolerância não acusaram nenhuma anormalidade na amostra.

Estabelecida a função discriminante, o próximo passo foi verificar se ocorreu agrupamento errado proposto pelo algoritmo da técnica do K-médias, em que a classificação originalmente feita foi tida como $100 \%$ corretas.

R. Árvore, Viçosa-MG, v.33, n.6, p.1149-1157, 2009

\section{CONCLUSÕES}

A relação negativa entre a renda e a DAC e a ausência de relação dela com o tamanho da propriedade, com base nos resultados desta pesquisa, são basicamente justificadas pelo viés da utilidade marginal. Assim, uma vez identificado o viés da utilidade marginal na amostra, afirma-se que a análise multivariada apresentou-se como método mais eficaz para se trabalhar com a valoração de contingente. A prova disso é que o método LOGIT não apresentou nenhuma relação estatisticamente significativa que permitisse obter média consistente da disposição a aceitar dos cafeicultores.

Caso o governo queira, portanto, fazer política pública que busque recuperar as áreas de matas, ele deveria dispor de uma quantia de aproximadamente de 54.000,00 reais por ano para recuperar uma área de 27 ha de mata, no caso dos cafeicultores relacionados com o cluster mais populoso (cluster 2). Já para os cafeicultores referentes ao cluster 4, o governo teria que despender $114.000,00$ reais por ano para recuperar 19 ha de mata nativa. Ou, então, para recuperar uma área de 46 ha, esses cafeicultores juntos estariam dispostos a aceitar 168.000,00 reais por ano. 
Para toda a população de cafeicultores do PROCAFÉ (82 cafeicultores), estima-se que 70 (86,5\%) fariam parte desses dois clusters, o que equivaleria a um dispêndio anual do governo de praticamente $\mathrm{R} \$ 254.200,00$. Ampliando essa análise para toda a população de cafeicultor de Viçosa, MG, estimada em 370 , provavelmente 314 teriam características similares aos dos clusters 2 e 4.

Assim, supõe-se que para viabilizar uma política pública o governo teria que despender, no total, 1.147.000,00 reais por ano, somente na região de Viçosa, para proporcionar crescimento das áreas de mata e, por sua vez, a provisão dos BSE.

\section{REFERÊNCIAS}

BISHOP, R. C.; HEBERLIN, T. A. Measuring values of extra-market goods: are indirect measures biased? American Journal of Agricultural Economics, v.61, n.5, p.926-930, 1979.

CHEE, Y. E. An ecological perspective on the valuation of ecosystem services. Biological Conservation, v.120, n.4, p.549-565, 2004.

COELHO, D. J. S.; SOUZA A. L.; OLIVEIRA, C. M. L. Levantamento da cobertura florestal natural da microrregião de Viçosa, MG, utilizando-se imagens de Landsat 5. Revista Árvore, v.29, n.1, p.17-24, 2005.

COOPER, P.; POE, G. L.; BATEMAN, I. J. The structure of motivation for contingent values: a case study of lake water quality improvement.

Ecological Economics, v.50, n.1, p.69-82, 2004.

GUIMARÃES, F. P. Intenção e ação conservacionista de proprietários rurais: aspectos gerais do conflito entre legislação ambiental e práticas agrícolas. Viçosa, MG: Universidade Federal de Viçosa, 2003. 35p. (Monografia).
HANEMANN, M. W. Welfare evaluation contingent valuation experiments with discrete response data. American Journal of Agricultural Economics, n.66, p.332-341, 1984.

\section{KREBS C. J. Ecological methodology. 2.ed. Menlo Park: Benjamin Cummings, 1998. 620p.}

LOOMIS, J. et al. Measuring the total economic value of restoring ecosystem services in an impaired river basin: results from a contingent valuation survey. Ecological Economics, v. 33, n.1, p.103-117, 2000.

MATTOS, A. D. M. et al. Valoração ambiental de áreas de preservação permanente da microbacia do Ribeirão São Bartolomeu no Município de Viçosa, MG. Revista Arvore, v.31, n.2, 2007. Disponível em: <http://www.scielo.br.> Acesso em: 18 de set. de 2007.

Mingoti, S. A. Análise de dados através de métodos de estatística multivariada: uma abordagem aplicada. Belo Horizonte: Universidade Federal de Minas Gerais, 2005. p.155-255.

SILVA, R.G. Valoração do Parque Ambiental "Chico Mendes", Rio Branco - AC: uma aplicação probabilística do método referendum com bidding games. 2003. 125f. Dissertação (Mestrado em Economia Aplicada) - Universidade Federal de Viçosa, Viçosa, MG, 2003.

SOUZA, A. L.; SOUZA, D. L. Análise multivariada para estratificação volumétrica de uma floresta ombrófila densa de terra firme, Amazônia Oriental. Revista Arvore, v.30, n.1, 2006. Disponível em: <http://www.scielo.br> Acesso em de out. de 2007. 
